\title{
The appropriateness of unbiased optical fractionators to assess cell proliferation in the adult hippocampus
}

\author{
Hamid R. Noori ${ }^{1,2} *$ and Casimir A. Fornal ${ }^{2}$ \\ 1 Institute of Psychopharmacology, Central Institute for Mental Health, University of Heidelberg, Mannheim, Germany \\ 2 Princeton Neuroscience Institute, Princeton University, Princeton, NJ, USA
}

\section{Edited by:}

Perry Bartlett, University of

Queensland, Australia

Reviewed by:

Maurice Curtis, Auckland University, New Zealand

Dhanisha Jhaveri, University of

Queensland, Australia

*Correspondence:

Hamid R. Noori, Institute of

Psychopharmacology, Central

Institute for Mental Health, University

of Heidelberg, J5, 68159 Mannheim,

Germany.

e-mail:hamid.noori@zi-mannheim.de
Optical fractionators have dominated the field of neural cell counting for two decades. These unbiased stereological techniques are often used for the quantification of hippocampal cell proliferation in neurogenesis experiments. However, the heterogeneous distribution of labeled cells, especially in the form of clusters, confounds the application of these techniques. A critical evaluation of the applicability of the optical fractionator suggests that absolute counting achieves higher efficiency in the quantification of cell proliferation than unbiased estimations.

Keywords: unbiased stereology, optical fractionator, cell counting, hippocampal cell proliferation
The discovery that new neurons are continuously produced throughout adulthood (adult neurogenesis) in discrete regions of the brain, notably within the dentate gyrus of the hippocampus and the olfactory bulb, has led to an explosion of studies aimed at elucidating the biological significance of this phenomenon as well as the factors that might impact this process. Neurogenesis is a complex, multi-step process, consisting of neural progenitor cell proliferation, differentiation, migration, neuronal maturation, and cell death (Ehninger and Kempermann, 2008). The production of new neurons can be modulated at multiple levels. Since cell proliferation represents the first critical step toward neurogenesis, many studies have focused primarily on this process or, at the very least, have included an examination of cell proliferation as part of a larger, comprehensive investigation on neurogenesis. Cell proliferation in these studies is usually measured by injecting the thymidine analog 5-bromo- $2^{\prime}$-deoxyuridine (BrdU), which becomes incorporated into newly synthesized DNA of cells during the $S$ phase of the cell cycle. After an appropriate post-injection survival period (e.g., $2 \mathrm{~h}$ ), animals are sacrificed and their brains are then processed for BrdU immunohistochemistry. Another common, reliable method for assessing cell proliferation is to stain for the endogenous nuclear protein Ki67, which is expressed during all phases of the active cell cycle $\left(\mathrm{G}_{1}, \mathrm{~S}, \mathrm{G}_{2}\right.$, and mitosis), but is absent during the resting $\left(G_{0}\right)$ phase (Kee et al., 2002). In the dentate gyrus of the hippocampus, newly proliferating cells are found predominantly in the subgranular zone (SGZ), where the neural progenitors reside, and often appear in clusters (Wojtowicz and Kee, 2006).

The optical fractionator combines two stereological components: a three-dimensional probe for counting cell nuclei, the optical dissector, and a sampling scheme, the fractionator. The optical dissector provides unbiased estimates of cell numbers independent of assumptions on the size and shape of the cells. Its value converges to the true number of cells as the number of samples increases. The fractionator involves sampling a known fraction of a structural component or region (e.g., the SGZ of the dentate gyrus). The number of cells $(N)$ is then estimated as:

$N=\frac{1}{\mathrm{tsf}} \times \frac{1}{\mathrm{asf}} \times \frac{1}{\mathrm{ssf}} \times \Sigma \mathrm{Q}^{-}$,

where $\Sigma \mathrm{Q}^{-}$is the total number of cells actually counted in the dissectors; asf, the area sampling fraction, is the area of the counting frame relative to the area associated with each $x, y$ movement; and ssf, the section sampling fraction, is the fraction of total sections sampled; and finally, the thickness sampling fraction tsf captures the part of the investigated cross-sectional area of the sampled sections (Gundersen et al., 1988; West et al., 1988, 1991, West and Gundersen, 1990). The optical fractionator has dominated the field of unbiased estimations of cell number and has been extensively used to quantify the number of cells in different brain structures. However, the application of this technique in neurogenesis studies is not rigorous.

Although this estimator is independent of assumptions on the size and shape of the cells, it is not free of assumptions about the structure of the tissue. Indeed, the efficiency of this technique depends on the homogeneity of the cell distribution in the region of interest. The distribution of proliferating cells in the SGZ of the hippocampus is not regular (Figure 1A). Depending on the size of the area of the counting frame (acf) of the dissector, there is a probability that the dissector might miss the proliferating cells. Furthermore, proliferating cells in the SGZ commonly appear in clusters and these clusters convert the approximately homogenous distribution of labeled cells in 
the hippocampus to a non-uniform and heterogeneous pattern (Figure 1B).

To circumvent the problem of the lack of homogeneity different strategies have been developed to improve the efficiency of the dissectors:

1) Enlargement of the acf to create an artificial homogeneity. The larger the acf the higher is the probability of finding the same number of proliferating cells in the acfs (Figure 1C). This fact most likely accounts for the similarities in the results between

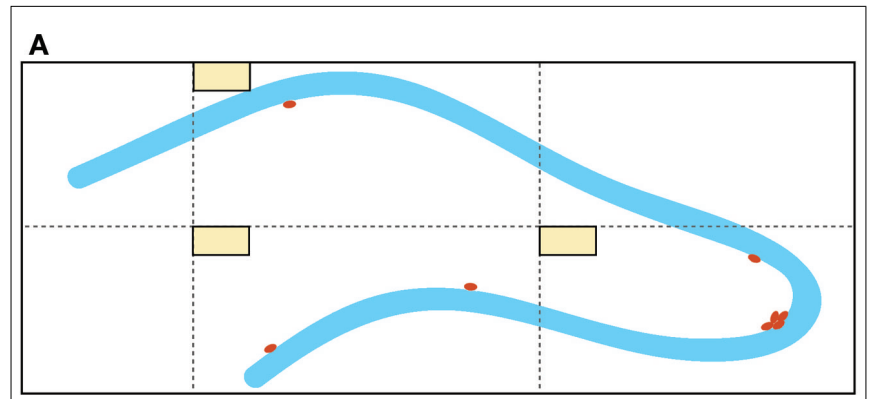

B

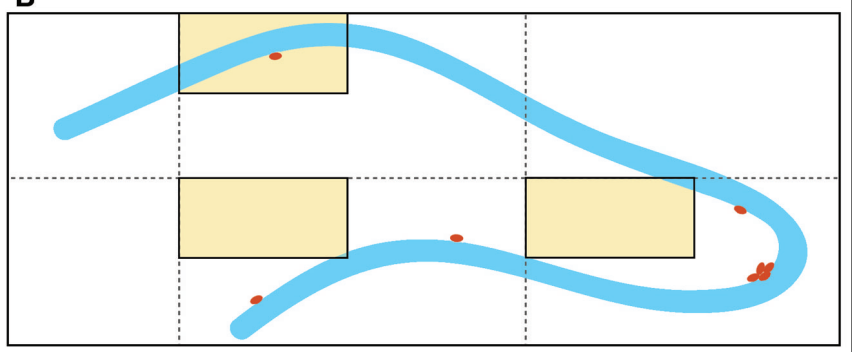

C

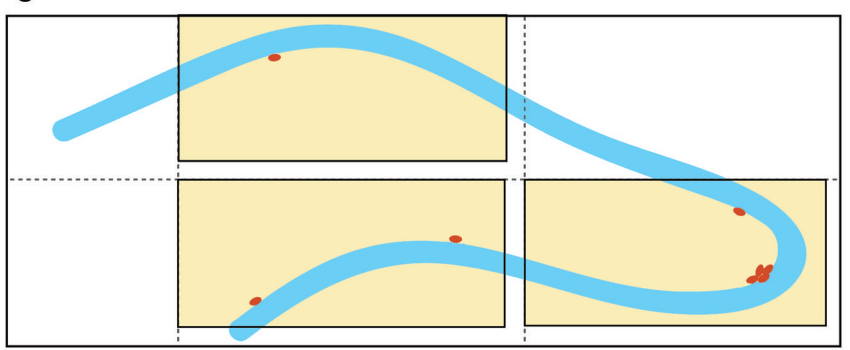

FIGURE 1 | Schematic representation of different sizes of the "area of the counting frame" (acf, shown as rectangular beige windows), superimposed on the dentate gyrus of the hippocampus (granular cell layer shown in blue and labeled proliferated cells depicted as brown dots). Enlargement of the acf by optical fractionators is a common method used to improve the accuracy of the quantification results. For example, in (A) none of the labeled cells fall within the small counting frames, whereas in (C), the larger counting frames capture all of the labeled cells, due to the irregular distribution of the labeled cells. Increasing the size of the acfs clearly gives better results, but the existence of labeled cell clusters (non-homogeneity) suggests a strong dependency between the size of the acfs and the efficacy of this technique [note that the cell cluster located near the apex of the dentate gyrus is captured by the counting frames in (C) but not (B)]. The large acfs depicted in (C) cover most of the area of the dentate gyrus and therefore, the counting results are comparable with absolute cell counting for the same area. absolute cell counting and application of the estimation techniques (Guzman-Marin et al., 2003; McCloskey et al., 2006, Almgren et al., 2007; Oomen et al., 2007). Nevertheless, these manipulations increase the value of the coefficient of error (CE) of the estimation. Unbiased does not imply error-free. Non-linear regression analysis of the stereological data (acf size $[50,150] \mu \mathrm{m}^{2}$, tissue volume $[0.09,1.38] \mathrm{mm}^{3}$, and estimated CE $[0.015,0.041])$ suggests a reciprocal functional relationship between the size of acfs and CEs:

$\mathrm{CE}(\mathrm{acf})=0.039 \times \mathrm{acf}^{-0.39}$.

The consequences of this model are considerable. This model shows that within the stereological design the smaller the size of acf the larger are the coefficients of error. Additionally, it suggests that the stereological coefficient of error is zero, if and only if the acf covers the whole tissue. These manipulations also abolish the major advantage of the dissector, which is to provide information on the number of cells for a large region, from the acfs, which are small pieces of the area of interest.

2) Novel modifications of the optical fractionator, the proportionator (Gardi et al., 2008a,b). These recently introduced methods use automatic image analysis to assign a weight proportional to some characteristic of the structure under study to each field of view in the section. Although these methods consider the heterogeneity of the tissue, they still have inherent problems. The proportionator requires a large number of cells to achieve a high efficiency; however, the number of proliferating cells labeled by BrdU (single injection) or Ki67 in neurogenesis studies is relatively low (approximately 20-60 cells/section) and depends strongly on the age of the animal at the time of sacrifice (Table 1). It should be noted that the utilization of more intensive BrdU regimens (such as BrdU in the drinking water over several days or weeks) results in a much larger proportion of BrdU-labeled cells in the dentate gyrus, which would be expected to improve stereology. However, these regimens may not provide an accurate assessment of cell proliferation, since a substantial number of newly labeled cells undergo apoptosis, and are subsequently rapidly cleared from the brain, due to the long survival period associated with the treatments. Therefore, the proportionator may not provide the optimal technique for the quantification of these cells. Moreover, the proportionator is a labor-intensive and expensive procedure.

Table 1 | Age-related decline in the number of Ki67-positive cells and clusters in the dentate gyrus of saline-treated male Sprague-Dawley rats ( $n=8$ /group).

\begin{tabular}{lccc}
\hline & 2 Month old & 31/2 Month old & 13 Month old \\
\hline Total cell density & $1397 \pm 74$ & $836 \pm 45$ & $212 \pm 14$ \\
Cluster density & $135 \pm 9$ & $82 \pm 8$ & $9 \pm 1$
\end{tabular}

Labeled cells/clusters were counted bilaterally and are expressed per cubic millimeter of tissue. Values are means \pm SEM. Data are from Cowen et al. (2008). Clusters were defined as in the caption to Figure 2. 

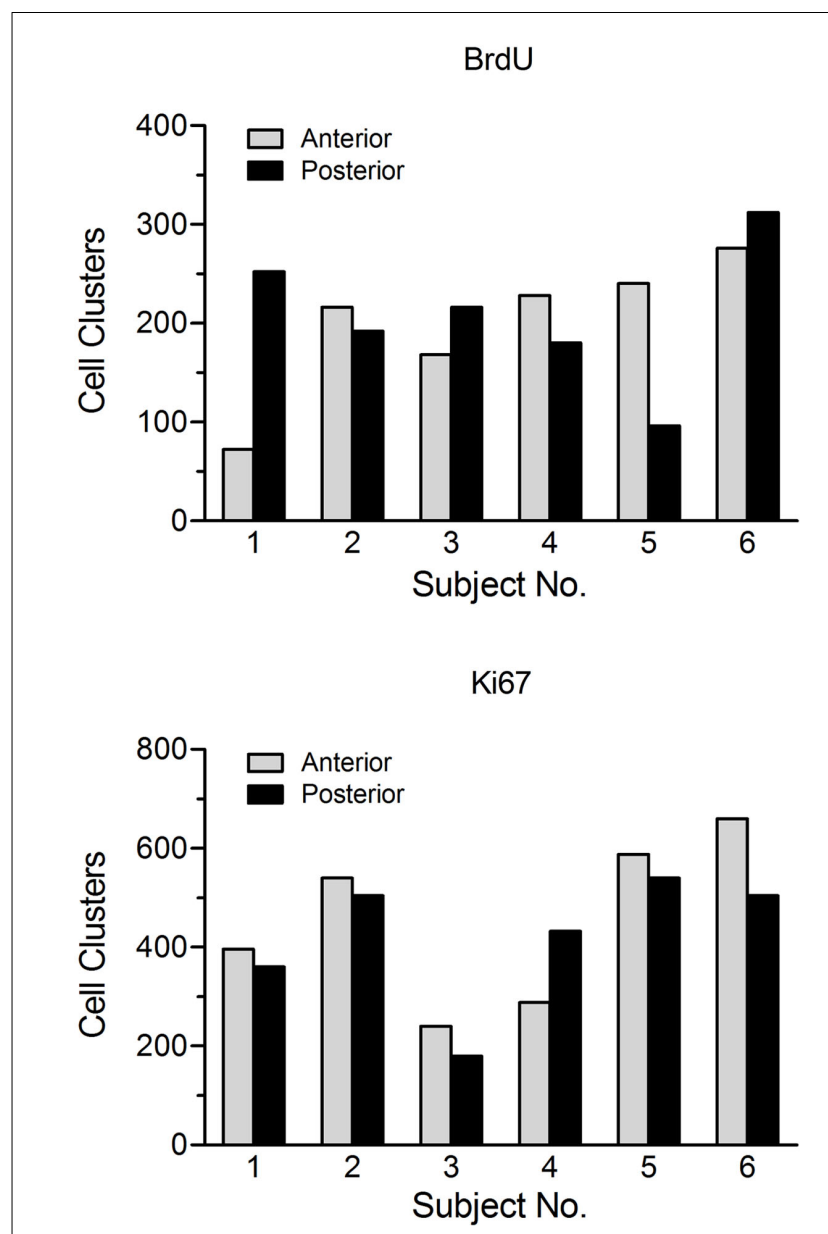

FIGURE 2 | Number of BrdU-positive and Ki67-positive cell clusters in the anterior (dorsal) versus posterior (ventral) dentate gyrus for a group of untreated adolescent ( $\sim \mathbf{6}$ week old) female Sprague-Dawley rats. This figure illustrates the large variability in both the total number and the regional distribution of labeled clusters within and between animals. Subjects were sacrificed $2 \mathrm{~h}$ after a single injection of BrdU $(200 \mathrm{mg} / \mathrm{kg}$, i.p.). Proliferating cells in the dentate gyrus were stained for both BrdU and the endogenous marker Ki67 using adjacent tissue sections from the same animal. For purposes of analysis, a cell cluster was defined as a grouping of three or more cells in close proximity $(\sim 5 \mu \mathrm{m})$ to each other.

3) Design-based stereology. The term design-based indicates that the methods and the sampling schemes used in stereology are defined a priori, in such a manner that one need not take into consideration the size, shape, spatial orientation, and especially spatial distribution of the cells to be quantitated (West, 2002). The major problem of applying these methods to cell proliferation studies relates to the main advantage of these techniques, i.e., the ability to define the spatial distribution of those cells in advance to design a new fractionator. The high variability in the regional distribution of BrdU- and Ki67-labeled cell clusters within and between animals (Figure 2) as well as the section to section differences in the cell counts for a given animal (Lagace et al., 2006) suggest that it is not possible to unify this information into a single (average) distribution function for hippocampal cell proliferation, which the design-based stereological technique relies on.

For quantification of BrdU- and Ki67-labeled cells in hippocampal proliferation studies it appears that unbiased stereological estimations offer little or no advantage over absolute cell counting, which has already been demonstrated in previous studies (Crews et al., 2004). Total cell counting performed bilaterally on every $6^{\text {th }}$ or $12^{\text {th }}$ section in the mouse or rat brain, respectively, by an experienced scorer usually takes about $30-45 \mathrm{~min}$ per animal and yields a small coefficient of error. Labeled cells can also be readily detected in the third dimension, i.e., section depth using the light microscope.

It should be emphasized that the above discussion does not address the overall efficiency of the techniques, but only in the case of quantifying the numbers of BrdU/Ki67-labeled cells in the SGZ. The optical fractionators have demonstrated high competency in applications of cell counting in relatively homogenous structures like the granule cell layer of the hippocampus.

\section{ACKNOWLEDGMENTS}

This work was supported by the Bundesministerium für Bildung und Forschung (NGFN Plus; FKZ: 01GS08152, FKZ: 01GS08155 see under www.ngfn-alkohol.de; FKZ: 01GS08151 and fundings from the Bernstein Center for Computational Neuroscience initiative; FKZ: 01GQ1003B), and Johnson \& Johnson Inc. We thank Professor Barry L. Jacobs (Princeton Neuroscience Institute) for his helpful comments.

image analysis and non-uniform probability proportional to size sampling. Comput. Biol. Med. 38, 313-328.

Gundersen, H. J. G., Bagger, P., Bendtsen, T. F., Evans, S. M., Korbo, L., Marcussen, N., Moller, A., Nielsen, K., Nyengaard, J. R., Pakkenberg, B., Sorensen, F. B., Vesterby, A., and West, M. J. (1988). The new stereological tools: disector, fractionator, nucleator and point sampled intercepts and their use in pathological reseach and diagnosos. APMIS 96, 857-881.

Guzman-Marin, R., Suntsova, N., Stewart, D. R., Gong, H., Szymusiak, R., and McGinty, D. (2003). Sleep deprivation reduces proliferation of cells in the dentate gyrus of the hippocampus in rats. J. Physiol. 549, 563-571.

Kee, N., Sivalingam, S., Boonstra, R., and Wojtowicz, J. M. (2002). The utility of Ki-67 and BrdU as proliferative markers of adult neurogenesis. J. Neurosci. Methods 115, 97-105.

Lagace, D. C., Yee, J. K., Bolanos, C. A., and Eisch, A. J. (2006). Juvenile administration of methylphenidate attenuates adult hippocampal neurogenesis. Biol. Psychiatry 60, 1121-1130. 
administration of methylphenidate attenuates adult hippocampal neurogenesis. Biol. Psychiatry 60, 1121-1130.

McCloskey, D. P., Hintz, T. M., Pierce, J. P., and Scharfman, H. E. (2006). Stereological methods reveal the robust size and stability of ectopic hilar granule cells after pilocarpineinduced status epilepticus in the adult rat. Eur. J. Neurosci. 24, 2203-2210.

Oomen, C. A., Mayer, J. L., de Kloet, E. R., Joels, M., and Lucassen, P. J. (2007). Brief treatment with the glucocorticoid receptor antagonist mifepristone normalizes the reduction in neurogenesis after chronic stress. Eur. J. Neurosci. 26, 3395-3401.

West, M. J. (2002). Design-based stereological methods for counting neurons. Prog. Brain Res. 135, 43-51.

West, M. J., Coleman, P. D., and Flood, D. G. (1988). Estimating the number of granule cells in the dentate gyrus with the dissector. Brain Res. 448, 167-172.

West, M. J., and Gundersen, H. J. G. (1990). Unbiased stereological estimation of the number of neurons in the human hippocampus. J. Comp. Neurol. 296, 1-22.

West, M. J., Slomianka, L., and Gundersen, H. J. G. (1991). Unbiased stereological estimation of the total number of neurons in the subdivisions of the rat hippocampus using the optical fractionator. Anat. Rec. 231, 482-497.

Wojtowicz, J. M., and Kee, N. (2006). BrdU assay for neurogenesis in rodents. Nat. Protoc. 1, 1399-1405.

Conflict of Interest Statement: The authors declare that the research was conducted in the absence of any commercial or financial relationships that could be construed as a potential conflict of interest.

Received: 22 September 2011; accepted: 05 December 2011; published online: 22 December 2011.
Citation: Noori HR and Fornal CA (2011) The appropriateness of unbiased optical fractionators to assess cell proliferation in the adult hippocampus. Front. Neurosci. 5:140. doi: 10.3389/fnins.2011.00140

This article was submitted to Frontiers in Neurogenesis, a specialty of Frontiers in Neuroscience.

Copyright (C) 2011 Noori and Fornal. This is an open-access article distributed under the terms of the Creative Commons Attribution Non Commercial License, which permits noncommercial use, distribution, and reproduction in other forums, provided the original authors and source are credited. 